\title{
Aging study on Resistive Plate Chambers of the CMS Muon Detector for HL-LHC
}

To cite this article: R. Aly et al 2020 JINST 15 C11002

View the article online for updates and enhancements.

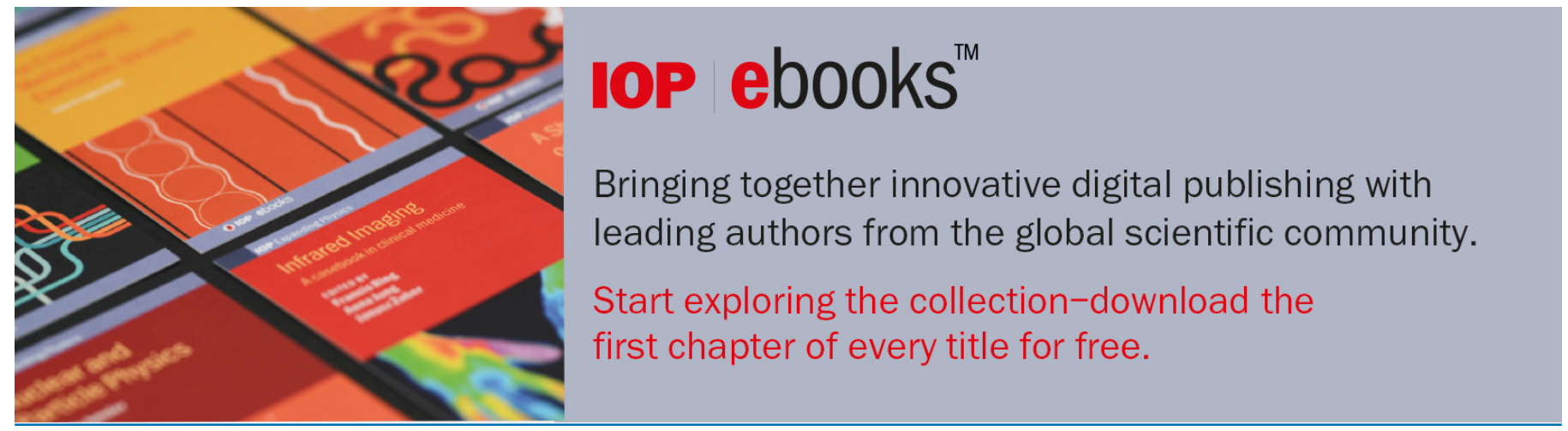




\section{Aging study on Resistive Plate Chambers of the CMS Muon Detector for HL-LHC}

R. Aly, ${ }^{n, 1}$ A. Gelmi, ${ }^{n}$ P. Kumari, ${ }^{l}$ N. Zaganidis, ${ }^{a}$ A. Samalan, ${ }^{a}$ M. Tytgat, ${ }^{a}$ G.A. Alves, ${ }^{b}$

F. Marujo, ${ }^{b}$ F. Torres Da Silva De Araujo, ${ }^{c}$ E.M. Da Costa,${ }^{c}$ D. De Jesus Damiao, ${ }^{c}$

H. Nogima, ${ }^{c}$ A. Santoro, ${ }^{c}$ S. Fonseca De Souza, ${ }^{c}$ A. Aleksandrov, ${ }^{d}$ R. Hadjiiska, ${ }^{d}$

P. laydjiev, ${ }^{d}$ M. Rodozov, ${ }^{d}$ M. Shopova, ${ }^{d}$ G. Sultanov, ${ }^{d}$ M. Bonchev, ${ }^{e}$ A. Dimitrov, ${ }^{e}$ L. Litov, ${ }^{e}$

B. Pavlov, ${ }^{e}$ P. Petkov, ${ }^{e}$ A. Petrov, ${ }^{e}$ S.J. Qian, ${ }^{f}$ C. Bernal, ${ }^{g}$ A. Cabrera, ${ }^{g}$ J. Fraga, ${ }^{g}$ A. Sarkar,${ }^{g}$

S. Elsayed, ${ }^{h}$ Y. Assran, ${ }^{b b, c c}$ M. El Sawy, ${ }^{b b}, d d$ M.A. Mahmoud, ${ }^{i}$ Y. Mohammed, ${ }^{i}$

C. Combaret,${ }^{j}$ M. Gouzevitch, ${ }^{j}$ G. Grenier,${ }^{j}$ I. Laktineh,${ }^{j}$ L. Mirabito ${ }^{j}$ K. Shchablo,${ }^{j}$

I. Bagaturia, ${ }^{k}$ D. Lomidze, ${ }^{k}$ I. Lomidze,${ }^{k}$ V. Bhatnagar, ${ }^{l}$ R. Gupta,,${ }^{l}$ J. Singh,,${ }^{l}$ V. Amoozegar, ${ }^{m}$

B. Boghrati, ${ }^{m, e e}$ M. Ebraimi, ${ }^{m}$ R. Ghasemi, ${ }^{m}$ M. Mohammadi Najafabadi, ${ }^{m}$ E. Zareian, ${ }^{m}$

M. Abbrescia, ${ }^{n}$ W. Elmetenawee, ${ }^{n}$ N. De Filippis, ${ }^{n}$ G. laselli, ${ }^{n}$ S. Leszki, ${ }^{n}$ F. Loddo, ${ }^{n}$

I. Margjeka, ${ }^{n}$ G. Pugliese, ${ }^{n}$ D. Ramos,${ }^{n}$ M. Caponero,,${ }^{f f}$ L. Benussi, ${ }^{o}$ S. Bianco, ${ }^{o}$

S. Colafranceschi, ${ }^{o}$ A. Russo, ${ }^{o}$ L. Passamonti, ${ }^{o}$ D. Piccolo, ${ }^{o}$ D. Pierluigi, ${ }^{o}$ G. Saviano, ${ }^{g} g$

S. Buontempo, ${ }^{p}$ A. Di Crescenzo, ${ }^{p}$ F. Fienga, ${ }^{p}$ G. De Lellis, ${ }^{p}$ L. Lista,${ }^{p}$ S. Meola, ${ }^{p}$

P. Paolucci, ${ }^{p}$ A. Braghieri, ${ }^{q}$ P. Salvini, ${ }^{q}$ P. Montagna, ${ }^{h h}$ C. Riccardi, ${ }^{h}$ P. Vitulo, ${ }^{h h}$

B. Francois, ${ }^{r}$ T.J. Kim, ${ }^{r}$ J. Park, ${ }^{r}$ S.Y. Choi, ${ }^{s}$ B. Hong, ${ }^{s}$ K.S. Lee,${ }^{s}$ J. Goh, ${ }^{t}$ H. Lee, ${ }^{u}$

J. Eysermans, ${ }^{v}$ C. Uribe Estrada, ${ }^{v}$ I. Pedraza, ${ }^{v}$ H. Castilla-Valdez, ${ }^{w}$ A. Sanchez-Hernandez, ${ }^{w}$

C.A. Mondragon Herrera, ${ }^{w}$ D.A. Perez Navarro, ${ }^{w}$ G.A. Ayala Sanchez, ${ }^{w}$ S. Carrillo, ${ }^{x}$

E. Vazquez, ${ }^{x}$ A. Radi, ${ }^{y}$ A. Ahmad, ${ }^{z}$ I. Asghar, ${ }^{z}$ H. Hoorani, ${ }^{z}$ S. Muhammad, ${ }^{z}$ M.A. Shah ${ }^{z}$ and I. Crotty ${ }^{a} a$ on behalf of the CMS Muon Group

${ }^{a}$ Ghent University, Department of Physics and Astronomy, Proeftuinstraat 86, B-9000 Ghent, Belgium

${ }^{b}$ Centro Brasileiro Pesquisas Fisicas,

R. Dr. Xavier Sigaud, 150 - Urca, Rio de Janeiro - RJ, 22290-180, Brazil

${ }^{c}$ Departamento de Fisica Nuclear e Altas Energias, Instituto de Fisica,

Universidade do Estado do Rio de Janeiro,

Rua Sao Francisco Xavier, 524, BR - Rio de Janeiro 20559-900, RJ, Brazil

${ }^{d}$ Bulgarian Academy of Sciences, Institute for Nuclear Research and Nuclear Energy,

Tzarigradsko shaussee Boulevard 72, BG-1784 Sofia, Bulgaria

${ }^{e}$ Faculty of Physics, University of Sofia,5 James Bourchier Boulevard, BG-1164 Sofia, Bulgaria

\footnotetext{
${ }^{1}$ Corresponding author.
} 
${ }^{f}$ School of Physics, Peking University, Beijing 100871, China

${ }^{g}$ Universidad de Los Andes, Apartado Aereo 4976, Carrera 1E, no. 18A 10, CO-Bogota, Colombia

${ }^{h}$ Egyptian Network for High Energy Physics, Academy of Scientific Research and Technology,

101 Kasr El-Einy St., Cairo, Egypt

${ }^{i}$ Center for High Energy Physics, Faculty of Science, Fayoum University, 63514 El-Fayoum, Egypt

${ }^{j}$ Université Lyon, Université Claude Bernard Lyon 1, CNRS/IN2P3, IP2I Lyon, UMR 5822,

F-69622, Villeurbanne, France

${ }^{k}$ Georgian Technical University, 77 Kostava Str., Tbilisi 0175, Georgia

${ }^{l}$ Department of Physics, Panjab University, Chandigarh 160 014, India

${ }^{m}$ School of Particles and Accelerators, Institute for Research in Fundamental Sciences (IPM),

P.O. Box 19395-5531, Tehran, Iran

${ }^{n}$ INFN, Sezione di Bari, Via Orabona 4, 70126 Bari, Italy

${ }^{\circ}$ INFN, Laboratori Nazionali di Frascati (LNF), Via Enrico Fermi 40, 00044 Frascati, Italy

${ }^{p}$ INFN, Sezione di Napoli, Complesso Univ. Monte S. Angelo, Via Cintia, 80126 Napoli, Italy

q INFN, Sezione di Pavia, Via Bassi 6, Pavia, Italy

${ }^{r}$ Hanyang University, 222 Wangsimni-ro, Sageun-dong, Seongdong-gu, Seoul, Republic of Korea

${ }^{s}$ Korea University, Department of Physics, 145 Anam-ro, Seongbuk-gu, Seoul 02841, Republic of Korea

${ }^{t}$ Kyung Hee University, 26 Kyungheedae-ro, Hoegi-dong, Dongdaemun-gu, Seoul, Republic of Korea

${ }^{u}$ Sungkyunkwan University,

2066 Seobu-ro, Jangan-gu, Suwon, Gyeonggi-do 16419, Seoul, Republic of Korea

${ }^{v}$ Benemerita Universidad Autonoma de Puebla, Puebla, Mexico

${ }^{w}$ Cinvestav,

Av. Instituto Politécnico Nacional No. 2508, Colonia San Pedro Zacatenco, CP 07360,

Ciudad de Mexico D.F., Mexico

${ }^{x}$ Universidad Iberoamericana, Mexico City, Mexico

${ }^{y}$ Sultan Qaboos University, Al Khoudh, Muscat 123, Oman

${ }^{z}$ National Centre for Physics, Quaid-i-Azam University, Islamabad, Pakistan

${ }^{a a}$ Department of Physics, Wisconsin University, Madison, WI 53706, United States

${ }^{b b}$ The British University in Egypt (BUE), Elsherouk City, Suez Desert Road, Cairo 11837, P.O. Box 43, Egypt

${ }^{c c}$ Suez University, Elsalam City, Suez - Cairo Road, Suez 43522, Egypt

${ }^{d d}$ Department of Physics, Faculty of Science, Beni-Suef University, Beni-Suef, Egypt

ee School of Engineering, Damghan University, Damghan, 3671641167, Iran

${ }^{\text {ff }}$ ENEA, Frascati, Frascati (RM), I-00044, Italy

${ }^{g g}$ Dipartimento di Ingegneria Chimica, Materiali e Ambiente, Sapienza Università di Roma, 00185, Roma, Italy

${ }^{h h}$ INFN, Sezione di Pavia and University of Pavia, Via Bassi 6, Pavia, Italy

E-mail: reham.aly@cern.ch

Aвstract: In the High Luminosity Large Hadron Collider (HL-LHC) program, during the next years, the instantaneous luminosity will increase up to $5 \times 10^{34} \mathrm{~cm}^{-2} \mathrm{~s}^{-1}$ which means a factor five higher than the nominal LHC luminosity. In that period, the present CMS Resistive Plate Chambers (RPC) system will be subjected to background rates higher than those for which the detectors have been designed, which could affect the detector properties and induce aging effects. To study whether the present RPC system can sustain the hard background conditions during the HL-LHC running 
period, a dedicated longevity test is ongoing at the CERN Gamma Irradiation Facility, where a few spare RPCs are exposed to high gamma radiation for a long term period to mimic the HL-LHC operational conditions. During the longevity test, the main detector parameters are continuously monitored as a function of the integrated charge. Preliminary results of the study, after having collected a sufficient amount of the expected integrated charge at HL-LHC, will be presented.

KeYwords: Gaseous detectors; Radiation-hard detectors; Resistive-plate chambers

ArXiv EPrint: 2005.11397 


\section{Contents}

1 Introduction 1

2 Aim of longevity study 1

3 Longevity test setup and procedure $\quad 2$

4 Detector parameter monitoring 3

4.1 Dark current and noise rate 3

4.2 Resistivity and current 4

5 Detector performance monitoring 5

6 Conclusion 6

\section{Introduction}

The Muon Tracking System, which lies on the outside of the Compact Muon Solenoid (CMS) experiment [1] at the CERN Large Hadron Collider (LHC), has been designed to provide an efficient muon trigger as well as a precise measurement of muon momentum and charge. It consists of three sub-detectors arranged in barrel and endcap regions: Drift Tubes in the barrel region, Cathode Strip Chambers in the endcap region and 1056 Resistive Plate Chambers (RPC) installed in both regions covering a pseudorapidity region up to $|\eta|=1.9$ [2, 3]. A CMS RPC chamber consists of two layers of $2 \mathrm{~mm}$ gas gaps with a sheet of segmented copper readout strips sandwiched between them and aligned along the direction parallel to the magnetic field lines. Each gas gap is made of two sheets of High-Pressure-Laminate (HPL) electrodes with $2 \mathrm{~mm}$ thickness and filled with a non-flammable three-component gas mixture of $95.2 \%$ freon $\left(\mathrm{C}_{2} \mathrm{H}_{2} \mathrm{~F}_{4}\right), 4.5 \%$ isobutane (i- $\mathrm{C}_{4} \mathrm{H}_{10}$ ), and $0.3 \%$ sulphur hexafluoride $\left(\mathrm{SF}_{6}\right)$ with a relative humidity of $\approx 40 \%$ [2]. The muon system worked efficiently during the LHC Runs I and II data takings at the nominal luminosity of $1 \times 10^{34} \mathrm{~cm}^{-2} \mathrm{~s}^{-1}[4-6]$.

\section{Aim of longevity study}

Gas detectors can suffer from aging effects when exposed to high radiation for long time which result in a degradation of detector performance appearing as loss in detector efficiency, increase in dark current ${ }^{1}$ and rise in noise rates. The main reason for this detector performance degradation are the chemical processes that happen inside the electron multiplication region where the gas

\footnotetext{
${ }^{1}$ Dark current is the current produced in the chamber when applying high voltage in the absence of background radiation.
} 
molecular fragments produced inside avalanches may form polymers growing on the electrodes' surface [7]. The present CMS RPC system has been certified for 10 years of LHC operation at a maximum background rate of $300 \mathrm{~Hz} / \mathrm{cm}^{2}$ and a total integrated charge of $50 \mathrm{mC} / \mathrm{cm}^{2}$. Based on the data collected by CMS during LHC Run II and assuming a linear dependence of the background rates as a function of the instantaneous luminosity, the expected background rates and integrated charge at HL-LHC will be about $600 \mathrm{~Hz} / \mathrm{cm}^{2}$ and $840 \mathrm{mC} / \mathrm{cm}^{2}$, respectively (including a safety factor of three) [8]. In those operating conditions a non-recoverable aging effects can appear, due to the higher collision rates and pile-up, ${ }^{2}$ that can affect the detector performance and properties. Therefore, a long term irradiation test has been carried out to study whether the present RPC detectors can survive the hard background conditions during the HL-LHC running period. During the longevity studies, the main detector parameters and performance are monitored as a function of the integrated charge to spot any possible aging effects.

\section{Longevity test setup and procedure}

A long term irradiation test has been started at the CERN Gamma Irradiation Facility (GIF++) which allows to test real size detectors. The GIF++ is a unique place equipped by a gamma source (13 TBq Cs-137) and a system of movable filters for varying the gamma flux which allow to test the detectors in a background condition similar to the ones at HL-LHC [9]. In addition to the gamma source, a $100 \mathrm{GeV}$ muon beam is provided 3-4 times per year for detector performance studies. The GIF++ facility provides also a controlled monitor of the environmental parameters during irradiation such as temperature and pressure. Four spare RPC chambers have been irradiated since July 2016, two RE2/2s and two RE4/2s [1,8] which are from the endcap region where the maximum background radiation rates are expected. The detectors are trapezoidally shaped with height $=1687 \mathrm{~mm}$, long side $=979 \mathrm{~mm}$ and short side $=684 \mathrm{~mm}$. Two different RPC production types have been tested, since the endcap RPC production was done in two different periods, i.e. all RPCs in endcap were done in 2005 except RE4/2 and RE4/3 which were made later in 2012/2013. Two chambers (one RE2 and one RE4) are continuously under irradiation while the other two chambers of the same type are kept as reference and switched on from time to time. All the detectors are flushed continuously with gas where the detectors are currently running with gas humidity $\approx 60 \%$ and 3 gas volume exchange per hour for irradiated chambers and one gas volume exchange per hour for reference chambers. The detector parameters (such as dark current, noise rate, current and count rates at several background conditions) are monitored continuously and compared with the measurements from the reference chambers to spot any degradation in the detector parameters due to long term irradiation. Moreover, when the muon beam at GIF++ is available, the detector performance is studied at different irradiation fluxes. Since the gamma flux is uniformly distributed over the detector surface, the integrated charge is calculated as the average density current accumulated in time in the three gaps that constitute the detector. The integrated charge collected from the beginning of irradiation till February 2020 are about 655 and $366 \mathrm{mC} / \mathrm{cm}^{2}$ for RE2 and RE4 chambers respectively as shown in figure 1 that correspond to approximately $78 \%$ and $44 \%$ of the expected integrated charges at HL-LHC.

${ }^{2}$ Pile-up known as more than one proton-proton collision takes place in the same beam crossing. 


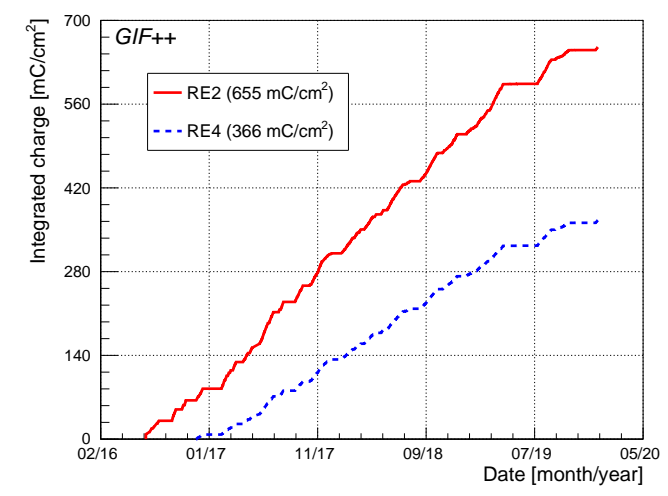

Figure 1. Integrated charge versus time, accumulated during the longevity test at GIF++ for RE2/2 (solid red line) and RE4/2 (dashed blue line) chambers. The RE4/2 chamber has been turned on a few months later because of total gas flow limitations. Different slopes account for different irradiation conditions during data taking.

\section{Detector parameter monitoring}

\subsection{Dark current and noise rate}

The dark current and noise rate are monitored periodically in order to spot any aging effect due to irradiation. The dark current density "Current normalized to the surface area" for RE2 both irradiated and reference chambers as a function of collected integrated charge is shown in figure 2. The dark currents were measured at $6.5 \mathrm{kV}$ (left), which represent the ohmic contribution, and at $9.6 \mathrm{kV}$ (right), which includes the gas amplification.
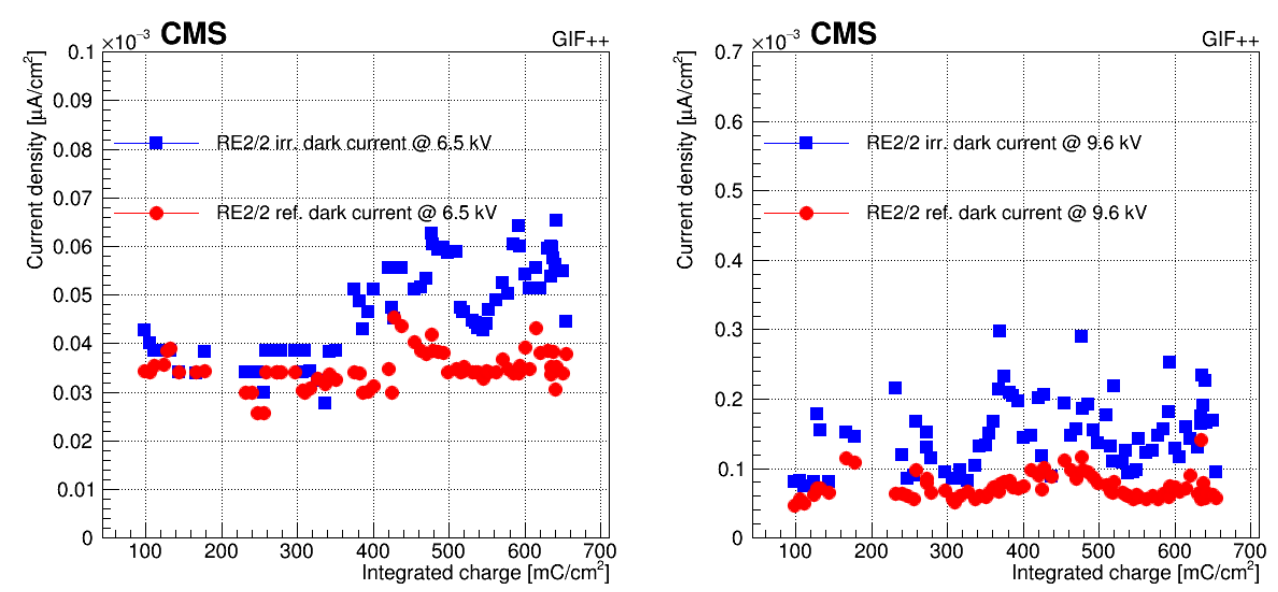

Figure 2. Dark current density for RE2 irradiated (blue squares) and reference (red circles) chambers as a function of collected integrated charge at $6.5 \mathrm{kV}$ (left) and at $9.6 \mathrm{kV}$ (right).

The dark current is almost stable in time with small acceptable variations of dark current level since the beginning of irradiation. Figure 3 (left) shows the dark current density monitored as function of effective high voltage (voltage normalized at the standard temperature $20^{\circ} \mathrm{C}$ and pressure 990 mbar [10]) at different values of collected integrated charge. The dark current is 
almost stable with time with small acceptable variations of dark current level since the beginning of irradiation. Figure 3 (right) shows the average noise rate for RE2 irradiated (blue) and reference (red) chamber as a function of collected integrated charge, the average noise rate is stable with time and less than $1 \mathrm{~Hz} / \mathrm{cm}^{2}$.
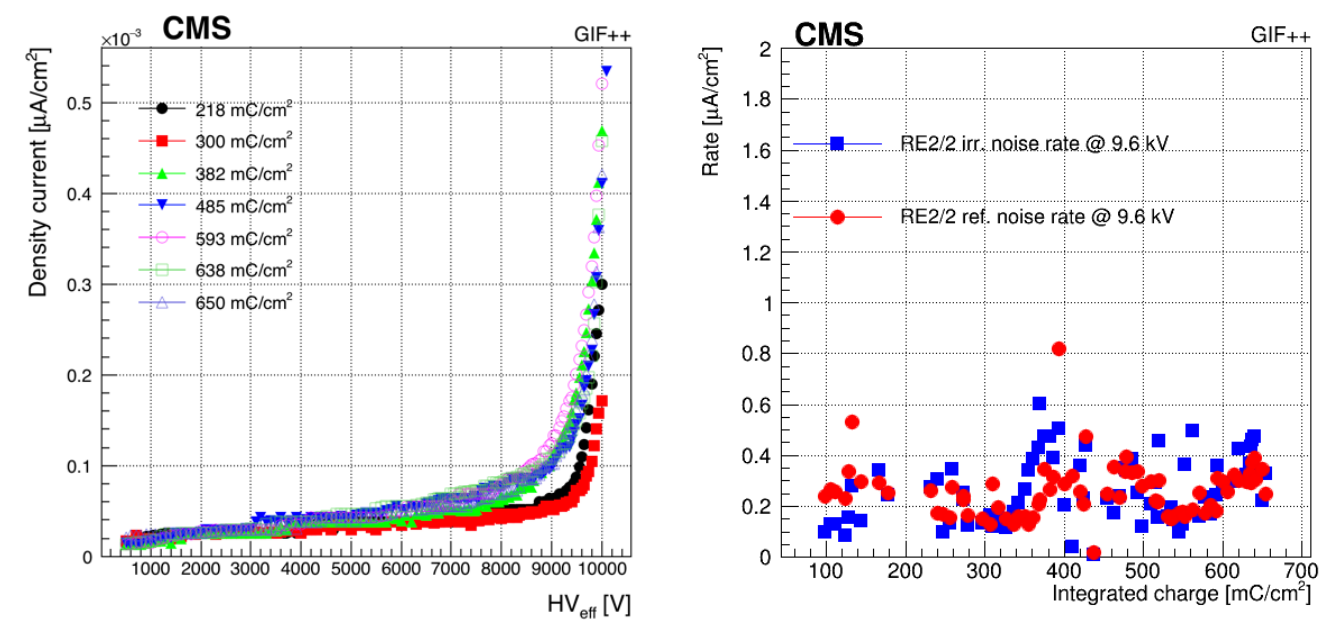

Figure 3. Dark current density monitored as a function of the effective high voltage at different values of collected integrated charge for RE2 irradiated chamber (left) and (right) average noise rate as a function of collected integrated charge for RE2 irradiated (blue squares) and reference chambers (red circles).

\subsection{Resistivity and current}

The current with the presence of background radiation is measured periodically as well. In addition, the electrode's resistivity is measured several times per year since it is a crucial parameter that influences the RPC performance. The resistivity is measured by filling up the detector with pure argon and operating in a self-sustaining streamer mode, when the gas quenching components such as the isobutane are removed the streamers propagate all over the detector area and by measuring the current and the applied high voltage the resistance can be calculated and hence the resistivity. The measured resistivity values are normalized to $20^{\circ} \mathrm{C}$ to allow comparing the values at different temperature conditions [11].

Figure 4 shows the resistivity ratio and the current ratio " current under gamma background rate of about $600 \mathrm{~Hz} / \mathrm{cm}^{2}$ " between irradiated and reference chambers, these ratios are taken to exclude the effect of external parameters. An increase in the resistivity was observed in the irradiated chamber in the first irradiation period, up to $\approx 300 \mathrm{mC} / \mathrm{cm}^{2}$, when the detectors operated in similar conditions as in CMS: one gas volume exchange per hour and $\approx 35-45 \%$ of relative gas humidity. These operating conditions were optimized for CMS, but they are not optimal with respect to the high gamma background rate $\left(\approx 600 \mathrm{~Hz} / \mathrm{cm}^{2}\right)$ at GIF++. Therefore, these conditions led to a drying up of the HPL plates with the consequent resistivity increase, which is also confirmed by the decrease of the currents. At $\approx 300 \mathrm{mC} / \mathrm{cm}^{2}$, the relative gas humidity was increased and maintained at $\approx 60 \%$, and the gas flow was increased in the irradiated chamber at three gas volume exchanges per hour. The combination of these effects allowed to reduce the HPL resistivity and mitigate the 
variations, proving that the observed resistivity increase was depending on the operating conditions and it is a recoverable effect.

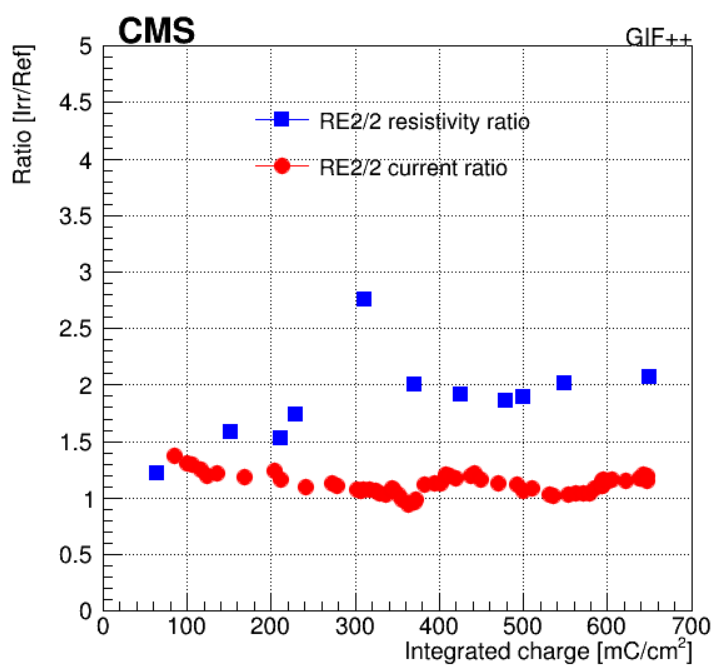

Figure 4. Resistivity ratio (blue squares) and current ratio (red circles) between RE2 irradiated and reference chambers as a function of collected integrated charge.

\section{Detector performance monitoring}

The detector performance has been measured during test beams before irradiation and at different periods of irradiation. The last measurement was done at $479 \mathrm{mC} / \mathrm{cm}^{2}$ at the last muon beam in GIF++ in 2018. Figure 5 shows the RE2 irradiated chamber efficiency measured as a function of the effective high voltage without background radiation (left) and in the presence of $600 \mathrm{~Hz} / \mathrm{cm}^{2}$ background (right) at different values of collected integrated charge. The efficiency is stable in time in the absence of the background radiation and we do not observe any working point shift [12], while in the case of presence of background, the efficiency is stable at the working point but we observe a working point shift of $100 \mathrm{~V}$ after collecting $260 \mathrm{mC} / \mathrm{cm}^{2}$ of integrated charge. The working point shift is related to the resistance $(R)$ of electrodes increase observed at $300 \mathrm{mC} / \mathrm{cm}^{2}$ of integrated charge as shown in figure 4. This increase of $R$ causes an increase of the voltage drop $(R I)$, where $I$ is the total current, on the effective voltage $(H V)$ applied to the electrodes, and the effective voltage on the gas $\left(H V_{\text {gas }}\right)$ is no longer the same [13,14]. The $H V_{\text {gas }}$ is defined as:

$$
H V_{\mathrm{gas}}=H V-R I
$$

where $R$ is the bakelite resistance and $I$ is the total current.

The detector operation regime is invariant with respect to $H V_{\text {gas }}$, therefore the efficiency as a function of $H V_{\text {gas }}$ does not depend anymore on the bakelite resistance as shown in figure 6 (left) which represents the efficiency at different irradiation periods and different background rates up to $600 \mathrm{~Hz} / \mathrm{cm}^{2}$. All the efficiency curves overlap and we do not observe anymore the working point shift, since the R increase effect on the electrodes has been removed. 

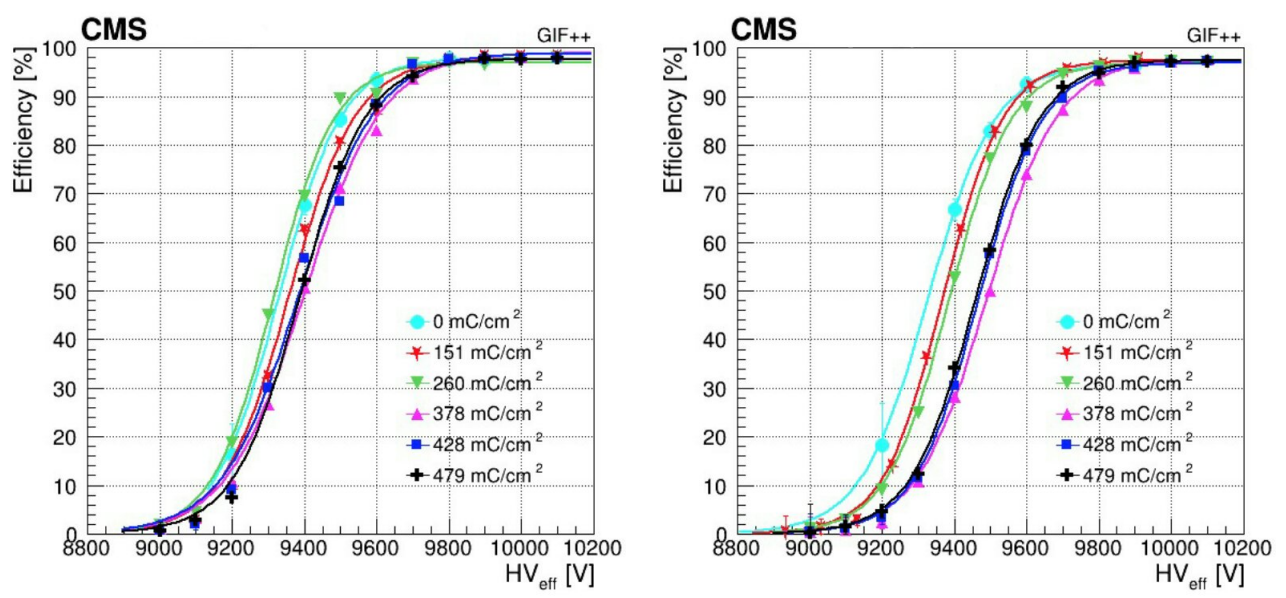

Figure 5. RE2/2 irradiated chamber efficiency as a function of the effective $H V$, with no irradiation (left) and under a gamma background rate of about $600 \mathrm{~Hz} / \mathrm{cm}^{2}$ (right) at different irradiation periods.
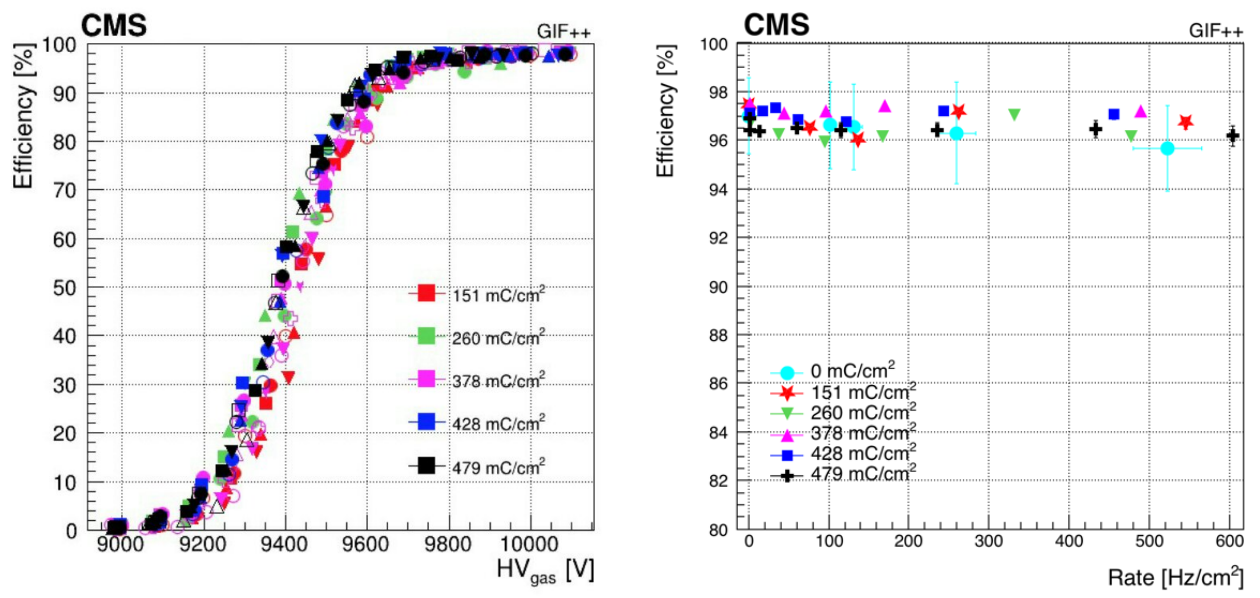

Figure 6. RE2/2 irradiated chamber efficiency as a function of the $H V_{\text {gas }}$ (left) at different background irradiation rates and different integrated charge values. The RE2 irradiated chamber efficiency at working point as a function of the background rate at different values of collected integrated charge (right).

The RE2 irradiated chamber efficiency at working point is measured at different background rates (up to $600 \mathrm{~Hz} / \mathrm{cm}^{2}$ ) and at different integrated charge values as shown in figure 6 (right). The efficiency is stable in time up to the highest background rate expected at HL-LHC $\left(600 \mathrm{~Hz} / \mathrm{cm}^{2}\right)$.

\section{Conclusion}

Longevity studies on spare resistive plate chambers are ongoing at the CERN Gamma Irradiation Facility under controlled conditions. Preliminary results show no evidence of any aging effect been observed so far. The main detector parameters and performance are stable. The integrated charge collected up to February 2020 represents $78 \%$ of the expected integrated charge at High Luminosity Large Hadron Collider, and more irradiation is needed to complete the study. 


\section{Acknowledgments}

We would like to thank our colleagues from CERN Gamma Irradiation facility where the measurements leading to those results have been performed with financial support from AIDA-2020 transnationale access program and INFN funds. This project has received funding from the European Union's Horizon 2020 Research and Innovation programe under Grant Agreement no. 654168. Also my sincere thanks to all CMS RPC members for their valuable work and RPC2020 organizers for a great conference.

\section{References}

[1] CMS collaboration, The CMS Experiment at the CERN LHC, 2008 JINST 3 S08004.

[2] CMS collaboration, The CMS muon project: Technical Design Report, Tech. Rep., CERN-LHCC-97-032, CMS-TDR-3, CERN, Geneva (1997).

[3] G. Pugliese, The RPC system for the CMS experiment, IEEE Nucl. Sci. Symp. Conf. Rec. 6 (2006) 822.

[4] CMS collaboration, The Performance of the CMS Muon Detector in Proton-Proton Collisions at $\sqrt{s}=7 \mathrm{TeV}$ at the LHC, 2013 JINST 8 P11002 [arXiv: 1306.6905].

[5] M.A. Shah et al., The CMS RPC Detector Performance and Stability during LHC RUN-2, 2019 JINST 14 C11012 [arXiv: 1808. 10488].

[6] CMS collaboration, Performance of the CMS muon detector and muon reconstruction with proton-proton collisions at $\sqrt{s}=13 \mathrm{TeV}, 2018$ JINST 13 P06015 [arXiv: 1804.04528].

[7] M. Abbrescia et al., HF production in CMS-Resistive Plate Chambers, Nucl. Phys. B Proc. Suppl. 158 (2006) 30.

[8] CMS collaboration, The Phase-2 Upgrade of the CMS Muon Detectors, Tech. Rep., CERN-LHCC-2017-012, CMS-TDR-016, CERN, Geneva (Sep, 2017).

[9] R. Guida, GIF++: A new CERN Irradiation Facility to test large-area detectors for the HL-LHC program, PoS ICHEP2016 (2016) 260.

[10] S. Colafranceschi et al., Resistive plate chambers for 2013-2014 muon upgrade in CMS at LHC, 2014 JINST 9 C10033.

[11] G. Carboni, S. De Capua, D. Domenici, G. Ganis, R. Messi, G. Passaleva et al., An extensive aging study of bakelite resistive plate chambers, Nucl. Instrum. Meth. A 518 (2004) 82.

[12] F. Thyssen, Performance of the resistive plate chambers in the CMS experiment, 2012 JINST 7 C01104.

[13] G. Pugliese et al., Aging studies for Resistive Plate Chambers of the CMS muon trigger detector, Nucl. Instrum. Meth. A 515 (2003) 342.

[14] G. Aielli, P. Camarri, R. Cardarelli, A. Di Ciaccio, L. Di Stante, B. Liberti et al., Further advances in aging studies for RPCs, Nucl. Instrum. Meth. A 515 (2003) 335. 